\title{
Deep topical anesthesia with ropivacaine-soaked sponge for phacoemulsification
}

\author{
Gianluca Scuderi $\cdot$ Luca Scuderi • Silvia Calafiore • Vito Fenicia • \\ Solmaz Abdolrahimzadeh
}

Received: 7 January 2019/Accepted: 22 May 2019/Published online: 31 May 2019

(C) The Author(s) 2019

\begin{abstract}
Purpose To assess safety and efficacy of deep topical anesthesia with ropivacaine-soaked sponge compared with topical anesthesia with oxybuprocaine in patients undergoing phacoemulsification.

Methods This was a retrospective study where records of patients operated for cataract were evaluated. Patients using a visual analogue scale scored pain during surgery, and the surgeon on a questionnaire recorded ease of operation. Medical records were evaluated for patients who received topical anesthesia with multiple administrations of oxybuprocaine $0.4 \%$ or those who received deep topical anesthesia with a polyvinyl acetal sponge impregnated with ropivacaine $0.75 \%$ and positioned under the eyelid $30 \mathrm{~min}$ before surgery.

Results A total of one hundred patient records, equally divided in patients receiving deep topical anesthesia or topical anesthesia, were included. The visual analogue scale scores among the groups were
\end{abstract}

G. Scuderi - S. Calafiore - V. Fenicia

S. Abdolrahimzadeh

Ophthalmology Unit, NESMOS Department, St. Andrea

Hospital, "Sapienza" University of Rome, Via di

Grottarossa 1035/1039, Rome, Italy

L. Scuderi $(\bowtie)$

Ophthalmology Unit, Department of Sense Organs,

Azienda Policlinico Umberto I, "Sapienza "University of

Rome, Viale del Policlinico 155, 00161 Rome, Italy

e-mail: lucascuderi@hotmail.com statistically significant for a lower pain score in patients who received deep topical anesthesia with ropivacaine-soaked sponges $(p=0.0069)$. The average surgeon score was significantly higher for the deep topical anesthesia group indicating favorable ease of surgery $(p=0.0341)$. Six patients had major complications during surgery. No additional anesthesia was necessary to manage the complications in four patients in the deep anesthesia group, whereas propofol was used for the induction and maintenance of anesthesia in two patients in the topical anesthesia group.

Conclusions Deep topical anesthesia with ropivacaine-soaked sponges performed as well as topical oxybuprocaine regarding safety and efficacy. It provided a lower patient pain score, favorable surgeon satisfaction, and long-lasting anesthesia.

Keywords Phacoemulsification - Topical anesthesia - Deep topical anesthesia - Ropivacainesoaked sponge - Pain score - Surgeon satisfaction · Cataract surgery

\section{Introduction}

The goals of any type of anesthesia in ophthalmic surgery are to provide pain free surgery, minimize risks of systemic complications, facilitate surgical procedure, and reduce risk of surgical complications. There are numerous options for anesthesia in cataract 
surgery, and anesthetic protocols have progressed from general anesthesia to topical techniques. Subtenon anesthesia has reduced the risk of complications of general anesthesia, but the technique is still associated with a possibility of complications of peribulbar/retrobulbar techniques $[1,2]$. In the latter methods, local anesthetic is delivered with a sharp needle in the spaces surrounding the eye and complications include globe perforation, damage to the optic nerve, retrobulbar hemorrhage, and ocular muscle injury [3-6]. The reduced need for akinesia due to evolving surgical techniques, advances in phacoemulsification instrumentation, reduction of incisional size for cataract extraction, and progression of anesthesia techniques, have played a major role to reach the faster, more controlled, and less traumatic cataract surgery of today.

Topical anesthesia for cataract surgery was introduced in 1992 and consists in the administration of anesthetic eye drops in the fornix before and during surgery [7]. Anesthesia is achieved by blocking the long and short ciliary nerves, the nasociliary nerve, and the lacrimal nerves, which are the afferent nerves of the cornea and the conjunctiva [7]. This method enables faster visual rehabilitation after surgery and is cost-effective. The technique eliminates the possible complications of injectable anesthesia, although it is more difficult for the surgeon as it requires a certain degree of cooperation from the patient [8]. Topical anesthesia does not eliminate pain sensitivity of the iris, zonule, and ciliary body. The intracameral technique of anesthesia was introduced in 1992. This technique is widely used with the intracameral administration of preservative free $1 \%$ lidocaine, which also contributes to mydriasis [9-12]. In 1995, Rosenthal developed a technique placing a lidocainesoaked sponge deep in the conjunctival fornix [13]. This deep, topical, "nerve-block" technique produced a level of anesthesia previously seen with injection techniques only [13]. In 1999, Koch-Assia introduced the use of xylocaine jelly for surface anesthesia $[14,15]$.

Today various agents are available for topical anesthesia like procaine, proparacaine, oxybuprocaine, tetracaine, lidocaine, prilocaine, bupivacaine, etidocaine and ropivacaine, which all have different times of onset and anesthetic duration [16-20]. Ropivacaine, recently introduced in clinical practice, couples a long anesthetic effect with a mild vasoconstrictive action. It is commonly chosen in place of shorter-lasting agents such as mepivacaine or lidocaine in an effort to extend the duration of postoperative analgesia, and to prolong surgical block. Ropivacaine is a new aminoamide, the first S-enantiomer local anesthetic in clinical use, that is known, to produce relatively less severe cardiotoxic and neurotoxic side effects, has wide margins of safety, [16-18], and is relatively safer on the corneal epithelium [21].

In the present study, we evaluated and compared the efficacy and safety of a ropivacaine-soaked sponge placed deep in the inferior fornix versus multiple topical drops of oxybuprocaine $0.4 \%$ in patients undergoing cataract surgery with phacoemulsification. The primary objectives were to assess patient pain during surgery and surgeon satisfaction. The secondary objectives were to assess adverse effects and complications attributable to the choice of anesthetic technique and the need for additional anesthesia during surgery.

\section{Methods}

Records of patients who underwent phacoemulsification for age-related cataract at the ophthalmology unit of the Sant'Andrea Hospital, "Sapienza" University of Rome, were evaluated over a 12-month period from 2016 to 2017. In the ophthalmology unit, in view of constant efforts to improve clinical service, all patients and surgeons complete questionnaires for cataract surgery for each operation. The records were evaluated for operations carried out by one surgeon (GS). Inclusion criteria were the presence of the red reflex, whereas exclusion criteria were patients with a history of inability to understand and follow verbal commands or those with mental status alterations, ocular disease other than cataract, and eyelid malformations.

The standard preparation protocol followed for cataract surgery in all patients was the administration of antibiotic eye drops for 3 days prior to the operation, pharmacological dilatation of the pupil for surgery with topical $0.5 \%$ tropicamide $+10 \%$ phenylephrine solution (one drop for 3 times, $1 \mathrm{~h}$ before surgery). In 2016, the surgeon used traditional topical anesthesia with one drop of topical oxybuprocaine $0.4 \%$ every $5 \mathrm{~min}$ for 4 times in the fornix for surgery. In 2017, all the operations were carried out with deep topical anesthesia where $30 \mathrm{~min}$ before 
surgery, a small $(1.5 \mathrm{~mm} \times 1.5 \mathrm{~mm})$ piece of polyvinyl acetal was cut, and dipped in ropivacaine $0.75 \%$ for $5 \mathrm{~min}$ and placed under the patient's eyelid in the inferior fornix. The sponge was removed immediately prior to surgery. All patients were monitored via electrocardiogram, noninvasive blood pressure measurement, and pulse oximetry by a certified anesthesiologist and nurse. Briefly, the surgical procedure was performed with a temporal clear corneal incision size of $2.8 \mathrm{~mm}$, followed by phacoemulsification with the stop and chop technique and implantation of an intraocular lens.

Fifteen minutes after surgery every patient graded the pain experienced during surgery using a visual analogue scale (VAS) from 0 (no pain) to 10 (unbearable pain). Immediately following surgery, the surgeon completed a modified questionnaire, originally conceived by Gupta et al. [22], which consisted of three items to evaluate patient cooperation, unwanted ocular movements, and anterior chamber stability. 1-3 points could be attributed to each item, and thus the, final possible surgeon score ranged from 3 to 9 for each operation. A higher grading indicated higher surgeon satisfaction.

The postsurgical conditions of the cornea and conjunctiva were evaluated with slip-lamp examination. Preoperative intraocular pressure (IOP) $30 \mathrm{~min}$ prior to surgery and postoperative IOP on the first postoperative day were measured with the Icare tonometer $\left(\operatorname{ICare}^{\circledR}\right.$ ), which does not require the instillation of anesthetic [23].

The study was conducted in accordance with the tenets of the Declaration of Helsinki and was given Institutional Review Board approval. All patients signed informed consent.

T-student and Mann-Whitney analyses were used to compare VAS score, surgeon score, and IOP measures. $p<0.05$ was considered statistically significant.

\section{Results}

One hundred eyes of 100 patients operated for cataract with phacoemulsification by one surgeon were included. Fifty consecutive patients operated using traditional topical anesthesia with oxybuprocaine in 2016 and 50 consecutive patients operated with deep topical anesthesia with ropivacaine-soaked sponges in
2017 were included. The groups were comparable for age, gender, surgery duration, and axial length. Although the number of right and left eyes in the two groups was different and surgery of the left eye can be more challenging, given that a temporal incision was performed in all cases, this did not affect the results. The average age of patients was 69.29 years (SD 6.60) (Table 1).

VAS score is reported in Table 2. The statistical difference between the VAS scores among the groups was significant $(p=0.0069)$ with a lower score for deep topical anesthesia indicating less reported pain during surgery. The average surgeon score was 5.7600 (SD 1.9646) in the topical anesthesia group and 6.5800 (SD 1.6548) in the deep topical anesthesia group (Table 2). Statistical analysis showed a significant difference between the two groups $(p=0.0341)$ with a higher surgeon score for deep topical anesthesia, implying better surgeon satisfaction.

Six patients had major complications during surgery; four had received deep topical anesthesia (2 posterior capsule ruptures and 2 iris prolapses), whereas two had received topical anesthesia (posterior capsule ruptures). In two of four cases in the deep topical anesthesia group, an anterior vitrectomy was performed. No additional anesthesia was necessary in this group. In the two patients in the topical anesthesia group, propofol was administered by the anesthetist for induction and maintenance of anesthesia in order to manage the complications.

Corneal epithelial alterations were not found in the postoperative examinations ( $24 \mathrm{~h}$ after surgery) in any of the patients. The difference between preoperative and postoperative IOP did not reflect a significant difference in the two groups ( $p=0.3919$, paired t-test) (Table 3). Three eyes received hypotensive treatment on the first postoperative day; two were treated with topical apraclonidine $0.5 \%$ and one received oral acetazolamide [24, 25].

\section{Discussion}

This institutional retrospective study demonstrated that both techniques of deep topical anesthesia with ropivacaine-soaked sponge and topical anesthesia with oxybuprocaine $0.4 \%$ provided good analgesia during phacoemulsification for cataract. The 
Table 1 Demographic data, surgery side, and axial length of patients undergoing topical versus deep topical anesthesia

\begin{tabular}{|c|c|c|c|c|c|c|c|c|}
\hline & \multicolumn{2}{|l|}{ Age } & \multicolumn{2}{|l|}{ Gender } & \multicolumn{2}{|c|}{ Surgery side } & \multicolumn{2}{|c|}{ Axial length } \\
\hline & Mean & SD & Female & Male & Right & Left & Mean & SD \\
\hline Tot & 69.29 & 6.60 & 58 & 42 & 52 & 48 & 22.15 & 1.8606 \\
\hline Topical & 68.60 & 6.79 & 30 & 20 & 17 & 33 & 22.02 & 1.7082 \\
\hline Deep topical & 69.98 & 6.39 & 28 & 22 & 35 & 15 & 22.28 & 2.0105 \\
\hline
\end{tabular}

Table 2 Visual analogue scale scores and surgeon satisfaction questionnaire scores in topical versus deep topical anesthesia groups

\begin{tabular}{|c|c|c|c|c|c|c|c|c|}
\hline & \multicolumn{2}{|l|}{ Tot } & \multicolumn{2}{|l|}{ Topical } & \multicolumn{2}{|c|}{ Deep Topical } & \multicolumn{2}{|c|}{$p$ value (two-sided) } \\
\hline & Mean & SD & Mean & SD & Mean & SD & t-student & Mann-Whitney \\
\hline VAS & 3.0000 & 2.1649 & 3.4800 & 2.1968 & 2.5200 & 2.0428 & 0.0259 & 0.0069 \\
\hline Surgeon questionnaire & 6.1700 & 1.8535 & 5.7600 & 1.9646 & 6.5800 & 1.6548 & 0.0263 & $0-0341$ \\
\hline
\end{tabular}

Table 3 Pre- and postoperative intraocular pressure in patients undergoing topical versus deep topical anesthesia

\begin{tabular}{|c|c|c|c|c|c|c|c|c|}
\hline & \multicolumn{2}{|l|}{ Tot } & \multicolumn{2}{|l|}{ Topical } & \multicolumn{2}{|c|}{ Deep Topical } & \multicolumn{2}{|c|}{$p$ value (two-sided) } \\
\hline & Mean & SD & Mean & SD & Mean & SD & $\begin{array}{l}\mathrm{t}- \\
\text { student }\end{array}$ & $\begin{array}{l}\text { Mann- } \\
\text { Whitney }\end{array}$ \\
\hline Preoperative IOP & 13.4600 & 2.7466 & 13.6000 & 2.6726 & 13.3200 & 2.8388 & 0.6127 & 0.4263 \\
\hline Postoperative IOP & 14.0900 & 2.8109 & 14.1000 & 2.7498 & 14.0800 & 2.8986 & 0.9718 & 0.9778 \\
\hline $\begin{array}{l}\text { Difference between pre- and postoperative } \\
\text { IOP }\end{array}$ & 0.6300 & 1.5086 & 0.5000 & 1.1824 & 0.7600 & 1.7792 & 0.3919 & 0.2150 \\
\hline
\end{tabular}

ropivacaine-soaked sponge method was equally safe and efficacious.

Based on the VAS scores, the patients who underwent deep topical anesthesia reported less pain. This is similar to the results of Martini et al. who reported a lower pain score in patients receiving topical ropivacaine with respect to topical lidocaine, although their results did not reach statistical significance [18], whereas Ugur et al. did not report any significant difference in pain score when comparing deep topical anesthesia with ropivacaine to deep topical anesthesia with lidocaine [26]. Similar to the results of Ugur [26], surgeon satisfaction was good in both groups, but in our study this was better in the deep topical anesthesia group.

Cataract surgery can be complicated, and surgery duration may be longer in eyes with pseudoexfoliation syndrome, glaucoma, floppy iris syndrome, high myopia or hyperopia, and other ocular conditions
$[27,28]$. Deep topical anesthesia using a sponge impregnated with ropivacaine provided sufficient and long-lasting analgesia that did not require supplemental anesthesia in any patient. Indeed, in the deep topical anesthesia group no additional anesthesia was necessary to manage complications, whereas additional propofol was required in the topical anesthesia group. Deep topical anesthesia seems to be a viable alternative to classic topical anesthesia especially in eyes with increased intraoperatory risks. Neither drug induced systemic and/or local toxicity; there were no corneal complications.

In conclusion, anesthesia with ropivacaine-soaked sponges for deep topical anesthesia appeared to be effective and safe and compared favorably with topical anesthesia. We acknowledge that our study had some limitations in that correlation assessment between the VAS and the surgeon questionnaire score was not performed. Furthermore, the response of the 
surgeon was not correlated with demographic criteria such as gender and age or ocular status such as cataract density. This will be a prerogative in future studies.

\section{Compliance with ethical standards}

Conflict of interest All authors declare that they have no conflict of interest.

Ethical approval All procedures performed in studies involving human participants were in accordance with the ethical standards of the institutional and/or national research committee and with the 1964 Helsinki declaration and its later amendments or comparable ethical standards.

Informed consent Informed consent was obtained from all individual participants included in the study.

Open Access This article is distributed under the terms of the Creative Commons Attribution 4.0 International License (http:// creativecommons.org/licenses/by/4.0/), which permits unrestricted use, distribution, and reproduction in any medium, provided you give appropriate credit to the original author(s) and the source, provide a link to the Creative Commons license, and indicate if changes were made.

\section{References}

1. Davidson M, Padroni S, Bunce C, Rüschen H (2007) SubTenon's anaesthesia versus topical anaesthesia for cataract surgery. Cochrane Database Syst Rev 3 CD006291

2. Rashmi S, Akshaya KM, Mahesha S (2014) Comparison of topical versus sub-Tenon's anesthesia in phacoemulsification at a tertiary care eye hospital. J Ophthalmic Vis Res 9:329-333

3. Cheng AC, Yuen HK, Lam RF, Lam DS (2005) SubTenon's block versus topical anaesthesia for cataract surgery. Br J Ophthalmol 89:1227-1228

4. Abelson MB, Mandel E, Paradis A, George M (1989) The effect of hyaluronidase on akinesia during cataract surgery. Ophthalmic Surg 20:325-326

5. Alhassan MB, Kyari F, Ejere HO (2008) Peribulbar versus retrobulbar anaesthesia for cataract surgery. Anesth Analg 107:2089

6. Sirinvasan S, Fern AI, Selvaraj S, Hasan S (2004) Randomized double-blind clinical trial comparing topical and sub-Tenon's anaesthesia in routine cataract surgery. Br J Anaesth 93:683-686

7. Fichman RA (1996) Use of topical anesthesia alone in cataract surgery. J Cataract Refract Surg 22:612-614

8. Studholme S (2008) A comparison of methods of local anaesthesia used for cataract extraction. J Perioper Pract 18:17-21

9. Westborg I, Mönestam E (2013) Intracameral anesthesia for cataract surgery: a population-based study on patient satisfaction and outcome. Clin Ophthalmol 7:2063-2068

10. Ezra DG, Nambiar A, Allan BD (2008) Supplementary intracameral lidocaine for phacoemulsification under topical anesthesia. A meta-analysis of randomized controlled trials. Ophthalmology 115:455-487

11. Nikeghbali A, Falavarjani KG, Kheirkhah A, Bakhtiari P, Kashkouli MB (2007) Pupil dilation with intracameral lidocaine during phacoemulsification. J Cataract Refract Surg 33(1):101-103

12. Fenicia V, Abdolrahimzadeh S, Scuderi G, Fabrizio L, Maurizi Enrici M, Cruciani F, Recupero SM (2015) Intracameral epinephrine without the addition of intracameral lidocaine in the management of tamsulosin associated intraoperative floppy iris syndrome. Clin Ter 166:158-161

13. Rosenthal KJ (1995) Deep, topical, nerve-block anesthesia. J Cataract Refract Surg 21:499-503

14. Chalam KV, Murthy RK, Agarwal S, Gupta SK, Grover S (2009) Comparative efficacy of tetraVisc versus lidocaine gel in cataract surgery. BMC Ophthalmol 9:7

15. Amiel H, Koch PS (2007) Tetracaine hdrocholoride $0.5 \%$ versus lidocaine $2 \%$ jelly as a topical anesthetic agent in cataract surgery: comparative clinical trial. J Cataract Refract Surg 33:98-100

16. Wang L, Shankarappa SA, Tong R, Ciolino JB, Tsui JH, Chiang HH, Kohane DS (2013) Topical drug formulations for prolonged corneal anesthesia. Cornea 32:1040-1045

17. Ramselaar JA, Boot JP, van Haeringen NJ, van Best JA, Oosterhuis JA (1988) Corneal epithelial permeability after instillation of ophthalmic solutions containing local anaesthetics and preservatives. Curr Eye Res 7:947-950

18. Martini E, Cavallini GM, Campi L, Lugli N, Neri G, Molinari P (2002) Lidocaine versus ropivacaine for topical anesthesia in cataract surgery. J Cataract Refract Surg 28:1018-1022

19. Gills JP, Rudisill JE (1974) Bupivacaine in cataract surgery. Ophthalmic Surg 5:67-70

20. Gills JP (2004) Effect of lidocaine on lens epithelial cells. J Catract Refract Surg 30:1153-1154

21. Penna EP, Tabbara KF (1986) Oxybuprocaine keratopathy: a preventable disease. Br J Ophthalmol 70:202-204

22. Gupta SK, Kumar A, Agarwal S (2010) Cataract surgery under topical anesthesia: gender-based study of pain experience. Oman J Ophthalmol 3:140-144

23. Scuderi GL, Cascone NC, Regine F, Perdicchi A, Cerulli A, Recupero SM (2011) Validity and limits of the rebound tonometer $\left(\right.$ ICare $\left.^{\circledR}\right)$ : clinical study. Eur J Ophthalmol 21:251-257

24. Scuderi GL, Romano MR, Perdicchi A, Cascone N, Lograno M (2008) Apraclonidine hydrochloride pharmacology and clinical use. Exp Rev Ophthalmol 3:149-153

25. Seuderi GL, Regine F, Perdicci A, Mannino G, Recupero SM (2006) Comparative efficacy of acetazolamide and apraclonidine in the control of intraocular pressure following phacoemulsification. Ophthalmologica 220:356-360

26. Ugur B, Dunder SO, Ogurlu M, Gezer E, Ozcura F, Gursoy F (2007) Ropivacaine versus lidocaine for deep-topical nerve-block anaesthesia in cataract surgery: a double-blind randomized clinical trial. Clin Exp Ophthalmol 35:148-151

27. Plateroti P, Plateroti AM, Abdolrahimzadeh S, Scuderi GL (2015) Pseudoexfoliation syndrome and pseudoexfoliation glaucoma: a review of the literature with updates on surgical management. J Ophthalmol 370371

28. Scuderi G, Contestabile MT, Scuderi L, Librando A, Fenicia V, Rahimi S (2018) Pigment dispersion syndrome and 
pigmentary glaucoma: a review and update. Int Ophthalmol 10:15-20. https://doi.org/10.1007/s10792-018-0938-7
Publisher's Note Springer Nature remains neutral with regard to jurisdictional claims in published maps and institutional affiliations. 